PROCEEDINGS OF THE

AMERICAN MATHEMATICAL SOCIETY

Volume 129, Number 10, Pages 3031-3035

S 0002-9939(01)05979-2

Article electronically published on March 29, 2001

\title{
STURM THEOREMS FOR DEGENERATE ELLIPTIC EQUATIONS
}

\author{
WALTER ALLEGRETTO
}

(Communicated by David S. Tartakoff)

\begin{abstract}
We employ a version of the Picone Identity, suitable for the $p$ Laplacean, to obtain a Sturm Comparison Theorem for generalized solutions. This answers a question posed by Dunninger in 1995 .
\end{abstract}

\section{INTRODUCTION}

The establishment of a Picone Identity has recently proved useful as a simple means of establishing a variety of results in Spectral Theory, Sturm Comparison Theorems, etc. for the $p$-Laplacean, $-\Delta_{p}$, and related equations. To the best of our knowledge, this version of the identity was first established by Dunninger [5], and exploited by Allegretto and Huang [3, 4, where several other references are given. More recent related arguments may be found in [2, [6], and [7].

In general terms, the results in the above references extend to $-\Delta_{p}$ results that had long been known for the ordinary Laplacean, i.e. for $p=2$. It is the purpose of this paper to continue in this direction.

The main motivation for this work is an open question mentioned in [5], and related to earlier results given in 11. Specifically, we first recall that a typical conclusion of a Sturm Theorem is that a solution $v$ of a differential equation (or inequality) cannot be positive in a domain $\Omega$. Employing Maximum Principle arguments, one then shows that $v$ must actually change sign in $\Omega$. The continuity of $v$ is important in these arguments. In [1, it was shown for $p=2$ and $v$ solution of an equation, that even if $v$ is merely assumed to be in $H^{1,2}$, then one could still conclude that $\mu(x \mid v(x) \geq 0)>0$ and $\mu(x \mid v(x) \leq 0)>0$. That such a conclusion may hold for $-\Delta_{p}$ is mentioned in [5] as an open question, which we answer. Furthermore, we investigate the set $\{x \mid v=0\}$ and show that while this set may have positive measure (under the degeneracy condition we give), it is possible to locate this set to some extent and to obtain conditions such that it has measure zero and thus obtain the conclusion $\mu(x \mid v(x)<0)>0, \mu(x \mid v(x)>0)>0$. These latter results thus also represent a modest extension of the linear result given in [1].

Received by the editors March 2, 2000.

2000 Mathematics Subject Classification. Primary 35B05; Secondary 35D99.

Key words and phrases. Picone's Identity, p-Laplacean, generalized solutions, Sturm Theorems.

Research supported in part by NSERC (Canada). 


\section{DEFINITIONS AND RESULTS}

Let $\Omega$ be a domain in $R^{n}$, possibly unbounded, and consider in $\Omega$ the formal expression $\ell$

$$
\ell(u)=-\nabla\left[A(x)|\nabla u|^{p-2} \nabla u\right]-a(x)|u|^{p-2} u .
$$

Here we assume $u \in H_{0}^{1, p}(\Omega) ; A(x) \geq 0 ; A(x), a(x) \in L^{\infty}(\Omega)$. Observe that we impose no regularity on $\partial \Omega$, nor boundedness of $\Omega$, nor uniform ellipticity of $\ell$.

Let $B_{\ell}(u, \varphi)$ be the form naturally associated with $\ell$. For $\varphi \in H_{0}^{1, p}(\ell)$ we set

$$
B_{\ell}(u, \varphi)=\int_{\Omega} A(x)|\nabla u|^{p-2} \nabla u \nabla \varphi-a(x)|u|^{p-2} u \varphi .
$$

Finally, let $\mathcal{L}$ denote the analogous expression

$$
\mathcal{L}(v)=-\nabla\left[B(x)|\nabla v|^{p-2} \nabla v\right]-b(x)|v|^{p-2} v
$$

with $B, b$ satisfying the same assumptions as $A, a$, and $B_{\mathcal{L}}$ denoting the associate expression.

We now state the following:

Theorem 1. Let $u \in H_{0}^{1, p}(\Omega), 0 \leq v \in H^{1, p}(\Omega)$ and assume $B_{\ell}(u, u) \leq 0$, $B_{\mathcal{L}}(v, \phi) \geq 0$ for any $\phi, 0 \leq \phi \in H_{0}^{1, p}(\Omega)$. If

$$
A \geq B, \quad a \leq b \quad \text { a.e. } \Omega,
$$

it follows that

$$
\text { (a) } \begin{aligned}
\int_{\Omega \cap\{v=0\}} b|u|^{p} \geq \int_{\Omega \cap\{v>0\}} B\left[|\nabla u|^{p}-p\left(\frac{|u|}{v}\right)^{p-1}|\nabla v|^{p-2} \nabla v \cdot \nabla u\right. \\
\left.+(p-1)\left(\frac{|u|}{v}\right)^{p}|\nabla v|^{p}\right]+\int_{\Omega \cap\{v=0\}} B|\nabla u|^{p}
\end{aligned}
$$

(b) If $p \geq 2$, then $\int_{\Omega} B|u|^{p}\left|\frac{\nabla v}{v+\varepsilon}\right|^{p} \leq D$ for some constant $D$, independent of $\varepsilon>0$.

Proof. Let $\varepsilon>0, \phi_{m} \in C_{0}^{\infty}(\Omega), 0 \leq \phi_{m} \rightarrow|u|$ in $H^{1, p}(\Omega)$, and without loss of generality pointwise a.e. Since $v \geq 0$, we recall the estimate given in [3], 4], and 5] which may be obtained by direct calculation

$$
\begin{aligned}
& B_{\mathcal{L}}\left(\phi_{m}, \phi_{m}\right)-B_{\mathcal{L}}(v\left.\frac{\phi_{m}^{p}}{(v+\varepsilon)^{p-1}}\right)+\int_{\Omega} b \phi_{m}^{p}\left[\frac{(v+\varepsilon)^{p-1}-v^{p-1}}{(v+\varepsilon)^{p-1}}\right] \\
& \geq \int_{\Omega} B\left[\left|\nabla \phi_{m}\right|^{p}-p\left(\frac{\left|\phi_{m}\right|}{v+\varepsilon}\right)^{p-1}|\nabla v|^{p-2} \nabla v \cdot \nabla \phi_{m}\right. \\
&\left.+(p-1)\left(\frac{\phi_{m}}{v+\varepsilon}\right)^{p}|\nabla v|^{p}\right] .
\end{aligned}
$$

We observe that the integrand on the right-hand side is nonnegative, by Young's Inequality. Here we have employed the fact that $\frac{\phi_{m}^{p}}{(v+\varepsilon)^{p-1}} \in H_{0}^{1, p}$ [8]. From 
assumption (3) we conclude

$$
\begin{aligned}
B_{\ell}\left(\phi_{m}, \phi_{m}\right)+\int_{\Omega} b \phi_{m}^{p} & {\left[\frac{(v+\varepsilon)^{p-1}-v^{p-1}}{(v+\varepsilon)^{p-1}}\right] } \\
\geq \int_{\Omega} B[ & \left|\nabla \phi_{m}\right|^{p}-p\left(\frac{\left|\phi_{m}\right|}{v+\varepsilon}\right)^{p-1}|\nabla v|^{p-2} \nabla v \cdot \nabla \phi_{m} \\
& \left.+(p-1)\left(\frac{\phi_{m}}{v+\varepsilon}\right)^{p}|\nabla v|^{p}\right]
\end{aligned}
$$

We note that $0 \leq \frac{(v+\varepsilon)^{p-1}-v^{p-1}}{(v+\varepsilon)^{p-1}} \leq 1$. Passing first to the limit as $m \rightarrow \infty$ in $(5)$ and then as $\varepsilon \rightarrow 0$ we obtain conclusion (a) by Fatou's Lemma and the Lebesque Convergence Theorem and by recalling that $\nabla v=0$ a.e. on $\{x \mid v(x)=0\}$. Conclusion (b) is immediate by taking the limit as $m \rightarrow \infty$ in (5). Indeed, the estimate given in [2], which may be obtained by direct calculation, for $p \geq 2$ gives

$$
\begin{aligned}
& \int_{\Omega} C_{p} B|\nabla| u|-| u\left|\frac{\nabla v}{v+\varepsilon}\right|^{p} \\
& \quad \leq \int_{\Omega} B\left[|\nabla| u||^{p}-p\left(\frac{|u|}{v+\varepsilon}\right)^{p-1}|\nabla v|^{p-2} \nabla v \cdot \nabla u+(p-1)\left(\frac{|u|}{v+\varepsilon}\right)^{p}|\nabla v|^{p}\right] \\
& \quad \leq \int_{\Omega \cap\{v=0\}} b|u|^{p}
\end{aligned}
$$

for some positive calculable constant $C_{p}$, independent of $v, \phi_{m}$, $\varepsilon$. The result is then immediate.

We have the following consequence in the uniform case.

Corollary 1. Assume further that if $\Omega_{0} \subset \subset \Omega$, then $u>\delta>0$ a.e. $\Omega_{0}$, for some $\delta=\delta\left(\Omega_{0}\right)$. If $B>0$ and $v \neq c u$ for some constant $c$, then $\mu(x \mid v \leq 0)>0$. Hence, if $\mathcal{L}(v)=0$, then $\mu(x \mid v \leq 0)>0$ and $\mu(x \mid v \geq 0)>0$.

Proof. Suppose not. Then $v>0$ a.e. $\Omega$ and thus from (a) of Theorem 1 we obtain $\nabla u=\frac{u}{v} \nabla v$ a.e. $\Omega$. Choose $\Omega_{0} \subset \subset \Omega$ and let $\psi_{m} \rightarrow v$ in $H^{1, p}\left(\Omega_{0}\right)$, where $\Omega_{0} \subset \subset \Omega$ and each $\psi_{m} \in H^{1, p}\left(\Omega_{0}\right) \cap L^{\infty}\left(\Omega_{0}\right)$. Without loss of generality, we may assume $0 \leq \psi_{m} \leq v$ and that the convergence is also pointwise. We observe that $\frac{\psi_{m}}{u} \rightarrow \frac{v}{u}$ in $L^{p}\left(\Omega_{0}\right)$ since $u$ is bounded away from zero in $\Omega_{0}$, and furthermore,

$$
\nabla\left(\frac{\psi_{m}}{u}\right)=\frac{\nabla \psi_{m}}{u}-\frac{\psi_{m}}{u^{2}} \nabla u=\frac{\nabla \psi_{m}}{u}-\frac{\psi_{m}}{v} \frac{\nabla v}{u} \rightarrow \frac{\nabla v}{u}-\frac{\nabla v}{u}=0
$$

in $L^{p}\left(\Omega_{0}\right)$, by the Lebesgue convergence theorem. We thus have $\nabla\left(\frac{v}{u}\right)=0$ in $\Omega_{0}$, i.e. $v=c u$ in $\Omega_{0}$ for some $c$. Since $\Omega_{0}$ is arbitrary, we conclude $v=c u$ in $\Omega$, and the result follows.

As mentioned earlier, Corollary 1 is the answer to the open question, mentioned in [5].

We now investigate what happens if we assume that $B, u$ are strictly positive in some specified $\Omega_{0} \subset \subset \Omega$, under further smoothness conditions on $\Omega_{0}$ and $p \geq 2$.

Corollary 2. Let $p \geq 2$ and $\Omega_{0} \subset \subset \Omega$ be a smooth subdomain of $\Omega$ such that $B>\delta_{1}>0$ and $u(x)>\delta_{1}>0$ a.e. in $\Omega_{0}$. If $v \geq 0$ in $\Omega$, then either $v=0$ a.e. in $\Omega_{0}$ or $\mu(x \mid v(x)=0) \cap \Omega_{0}=0$. Consequently, if $B>\delta_{1}>0, u>\delta_{1}>0$ in any smooth domain $\Omega_{i} \subset \subset \Omega$ for some $\delta_{1}=\delta_{1}\left(\Omega_{i}\right)>0$, with $\Omega=\bigcup \Omega_{i}$ and $\Omega_{i} \subset \subset \Omega_{i+1}$, then $\mathcal{L} v=0$ implies either $v=c u$ or $\mu(x \mid v>0)>0$ and $\mu(x \mid v<0)>0$. 
Proof. Theorem 1(b) implies that

$$
\int_{\Omega_{0}}\left|\nabla\left(\ln \left(\frac{v+\varepsilon}{\varepsilon}\right)\right)\right|^{p}
$$

is bounded uniformly of $\varepsilon$. If $\mu(x \mid v=0) \cap \Omega_{0}>0$, then $\int_{\Omega_{0}}\left|\ln \left(\frac{v+\varepsilon}{\varepsilon}\right)\right|^{p}$ is also bounded independently of $\varepsilon$, as a consequence of [8, p. 60] and the assumed smoothness of $\Omega_{0}$. Without loss of generality, we then have $\ln \left(\frac{v+\varepsilon}{\varepsilon}\right) \rightarrow \psi$ in $L^{p}\left(\Omega_{0}\right)$ as $\varepsilon \rightarrow 0$. But $\ln \left(\frac{v+\varepsilon}{\varepsilon}\right) \rightarrow+\infty$ on the set $\{x \mid v(x)>0\}$ and the first result follows. Next, we have from Corollary 1 that $\mu(x \mid v(x) \leq 0)>0, \mu(x \mid v(x) \geq 0)>0$. Suppose $\mu(x \mid v(x)=0)>0$ and $v(x) \geq 0$. Either $v \equiv 0$ (i.e. $v=c u$ with $c=0$ ) or else we find an $\Omega_{i}$ in which $\mu(x \mid v>0)>0$ and $\mu(x \mid v=0)>0$, contradicting the first result of this corollary.

We conclude with an example that shows that the situation $u \geq 0, v \geq 0$, $\mu(x \mid u(x)=0)>0, \mu(x \mid v(x)=0)>0, v \neq c u$ can actually happen if $B$ is allowed to be zero, but as given by Corollary 2, either $v=0$ or $v>0$ in regions where $B>0, u>0$.

Example 1. Let $\Omega=(-1,1)$ and put

$$
\begin{aligned}
A=B & = \begin{cases}x^{p} & 0 \leq x<1, \\
\alpha(x) & -1<x<0 ;\end{cases} \\
a=b & = \begin{cases}-2^{p-1}(2 p-1) & 0 \leq x<1, \\
\beta(x) & -1<x<0 ;\end{cases} \\
v & = \begin{cases}\frac{x^{2}}{2} & 0 \leq x<1, \\
0 & x<0 .\end{cases}
\end{aligned}
$$

where $\alpha(x), \beta(x)$ are to be chosen as indicated below. Note first that $-\left(A\left|v^{\prime}\right|^{p-2} v^{\prime}\right)^{\prime}$ $-a|v|^{p-2} v=0$ in $\Omega$, regardless of $\alpha, \beta$. Now choose $\alpha, \beta, 0 \leq u \in H_{0}^{1, p}(\Omega)$ such that $B_{\ell}(u, u) \leq 0$. We then have $u \geq 0, v \geq 0, \mu(x \mid v(x)=0)>0$ and $v \neq c u$; furthermore, in regions where $A$ and $u$ are positive, then $v \equiv 0$ or $v>0$.

\section{REFERENCES}

[1] W. Allegretto, A comparison theorem for nonlinear operators, Annali Scuola Normal Sup. Pisa 25 (1971), 41-46. MR 45:7233

[2] W. Allegretto, Sturm type theorems for solutions of elliptic nonlinear problems, Nonlinear Differential Equations and Applications (to appear).

[3] W. Allegretto and Y.-X. Huang, A Picone's identity for the $p$-Laplacean and applications, Nonlinear Analysis T.M.A. 32 (1998), 819-830. MR 99c:35051

[4] W. Allegretto and Y.-X. Huang, Principal eigenvalues and Sturm comparison via Picone's identity, J. Differential Equations 156 (1999), 427-438. MR 2000g:35164

[5] D.R. Dunninger, A Sturm comparison theorem for some degenerate quasilinear elliptic operators, Boll. U.M.I. 9-A (1995), 117-121. MR 96b:35011

[6] J. Fleckinger, E.M. Harrell and F. de Thelin, Boundary behavior and estimates for solutions of equations containing the p-Laplacean, Electronic J. Differential Equations 1999 (1999), 1-19. MR 2000k:35083 
[7] J. Jaros and T. Kusano, A Picone type identity for second order half-linear differential equations, Acta Math. Univ. Comenianae 68 (1999), 137-151. MR 2000h:34060

[8] G.M. Troianiello, Elliptic Differential Equations and Obstacle Problems, Plenum Press, New York, 1987. MR 92b:35004

Department of Mathematical Sciences, University of Alberta, Edmonton, Alberta, Canada T6G 2G1

E-mail address: wallegre@retl.math.ualberta.ca 